\title{
Quantum phase properties of two-mode Jaynes-Cummings model for Schrödinger-cat states: interference and entanglement
}

\author{
Faisal A. A. El-Orany, ${ }^{1}$ M. H. Mahran, ${ }^{2}$ and M. R. B. Wahiddin and A. M. Hashim ${ }^{3}$ \\ 1 Department of Mathematics and Computer Science, \\ Faculty of Science, Suez Canal University, Ismailia, Egypt \\ ${ }^{2}$ Faculty of Computers \& Informatics, Suez Canal University, Ismailia, Egypt \\ ${ }^{3}$ Centre for Computational and Theoretical Sciences, Kulliyyah of Science, \\ International Islamic University Malaysia, 53100 Kuala Lumpur, Malaysia
}

(Dated: August 22, 2018)

\begin{abstract}
In this paper we investigate the quantum phase properties for the coherent superposition states (Schrödinger-cat states) for two-mode multiphoton Jaynes-Cummings model in the framework of the Pegg-Barnett formalism. We also demonstrate the behavior of the Wigner $(W)$ function at the phase space origin. We obtain many interesting results such as there is a clear relationship between the revival-collapse phenomenon occurring in the atomic inversion (as well as in the evolution of the $W$ function) and the behavior of the phase distribution of both the single-mode and two-mode cases. Furthermore, we find that the phase variances of the single-mode case can exhibit revival-collapse phenomenon about the long-time behavior. We show that such behavior occurs for interaction time several times smaller than that of the single-mode Jaynes-Cummings model.

PACS numbers: $42.50 \mathrm{Dv}, 42.60 . \mathrm{Gd}$
\end{abstract}

\section{INTRODUCTION}

One of the few exactly solvable fully quantum-mechanically models describing the interaction between the single-mode electromagnetic wave and two-level atom is the Jaynes-Cummings model JCM [1]. Various nonclassical effects have been reported for this system, in particular, when the field is initially prepared in the coherent light. For instance, the revival-collapse phenomenon (RCP) in the atomic inversion [2], quadrature squeezing [3] and sub-Poissonian statistics [4]. Furthermore, JCM has been used as a source for generating nonclassical states in the framework of the conditional measurement technique [5]. For more details related to JCM reader can consult the review paper, e.g., 6]. Actually, this simple model, i.e. single-mode interacts with two-level atom, has been extended to include multimode fields [7], multilevel atoms [8], multiatom interactions [9], and damping [10]. On the other hand, several experiments have been performed for the JCM, 
e.g., isolated single atoms [11], a single-mode two-photon Rydberg atom "micromaser" [12] and observation of RCP in the evolution of the atomic inversion [13].

The progress in the generation of quantum mechanical states, e.g. in a trapped ion [14], nondemolation measurement [15], conditional measurement [5], ..etc., encourages researchers to analyse the behavior of the JCM for these states. One of these states is the Schrödinger-cat state [16], which can exhibit various of the nonclassical effects arising from the interference in phase space. These states can be represented (for the $j$ th mode of convenience) as

$$
\begin{aligned}
& |\alpha\rangle_{\epsilon_{j}}=N_{j}\left[\left|\alpha_{j}\right\rangle+\epsilon_{j}\left|-\alpha_{j}\right\rangle\right], \\
& =\sum_{n=0}^{\infty} C_{n}^{(j)}|n\rangle
\end{aligned}
$$

where $\left|\alpha_{j}\right\rangle$ is a coherent state of the $j$ th mode with complex amplitudes $\alpha_{j} ; \epsilon_{j}$ is a c-number takes on values, $0,1,-1$ denoting coherent states, even coherent states and odd coherent states. Also $N_{j}$ is the normalization constant having the form

$$
N_{j}^{2}=\frac{1}{\left[1+\epsilon_{j}^{2}+2 \epsilon_{j} \exp \left(-2\left|\alpha_{j}\right|^{2}\right)\right]} .
$$

In the second line of (11) we have expressed the states as a linear combination of Fock states, where the probability distribution amplitudes are

$$
C_{n}^{(j)}=N_{j} \exp \left(-\frac{1}{2}\left|\alpha_{j}\right|^{2}\right) \frac{\alpha_{j}^{n}}{\sqrt{n !}}\left[1+(-1)^{n} \epsilon_{j}\right]
$$

Throughout this paper we use the shorthand notation $C_{n_{j}}^{(j)}$ instead of $C_{n_{j}}^{(j)}\left(\epsilon_{j}\right)$. The two-mode density matrix of the cat state (10), which includes 16 elements, can be briefly written as

$$
\begin{array}{r}
\hat{\rho}_{f}(0)=|\alpha\rangle_{\epsilon}|\alpha\rangle_{\epsilon^{\prime} \epsilon^{\prime}}\left\langle\left.\alpha\right|_{\epsilon}\langle\alpha|\right. \\
=\hat{\rho}_{I}+\hat{\rho}_{M},
\end{array}
$$

where $\hat{\rho}_{M}$ and $\hat{\rho}_{I}$ are the statistical-mixture and interference parts whose exact forms are given in 17]. As we mentioned above that for the single-mode cat states the origin of the occurrence of the nonclassical effects is in the quantum interference between the components of the states. For instance, coherent states are close to classical states, however, even (odd) coherent states exhibit significant squeezing (sub-Poissonian statistics) and their photon number distributions provide 
oscillatory behavior. It is worth reminding that the evolution of the Schrödinger-cat states with the single-mode JCM has been treated in [18, 19, 20, 21].

The phase properties of the JCM using the Pegg-Barnett formalism [22] have been investigated in, e.g. [23, 24, 25], in particular, when the field is initially prepared in the coherent state. It has been shown that for the single-mode JCM the evolution of both the phase variance and the phase distribution can carry certain information on the RCP of the corresponding atomic inversion [23]. Moreover, the phase variances of the multiphoton single-mode JCM exhibit RCP in the course of the long-time interaction [24]. Recently, the phase properties of the two-mode JCM (TMJCM) in the form of linear interaction rather than nonlinear version is studied in [26]. In the present paper we investigate the phase properties of the TMJCM whose Hamiltonian is described by the multiphoton hyper-Raman process and the modes are initially prepared in the Schrödingercat states (4). We use Pegg-Barnett formalism to perform such investigation. There are various mechanisms controlling the evolution of the considered system, e.g., interference in phase space, entanglement between the two modes (boson-boson interaction) as well as between the two modes and the two-level atom. The aim of the present analysis is to investigate the influence of these mechansims on the behavior of the phase distribution. Needless to say that the dynamics of the system are more complicated than those of the single-mode JCM. Moreover, such investigation is motivated by several facts. For instance, entanglement is connecting with the quantum computers and quantum cryptography [27]. Additionally, decoherence is at the heart of quantum measurement theory [28]. Also the phase distribution has been realised experimentally by means of the optical homodyne tomography [29], which allows quantum phase mean values to be calculated via the measured field density matrix.

In this paper we obtain many interesting results for TMJCM. For example, there is a good connection between the behavior of the atomic inversion and that of the corresponding phase distribution of both the single-mode and compound-mode cases. Also we show that under certain condition there is a relationship between the evolution of the atomic inversion and the $W$ function at the phase space origin. Furthermore, the evolution of the phase variance of the single-mode case can exhibit RCP about the long-time behavior, which occurs on the time domain several times smaller than that of the single-mode JCM. We analyse the behavior of the TMJCM in the following order: In section 2 we give the basic equations and relations, which will be used in the paper. Section 3 is devoted to discuss the main results. Summary of the results is given in section 4. 


\section{BASIC EQUATIONS AND RELATIONS}

In this section we describe the system and provide the main relations used in the paper. Such relations include the Hamiltonian of the system, the corresponding wave function and the calculations associated with the Pegg-Barnett formalism for the system [22].

We start with the Hamiltonian, which describes the interaction of the two modes with the single two-level atom and can briefly be written as

$$
\frac{\hat{H}}{\hbar}=\hat{H}_{f}+\hat{H}_{I}
$$

where $\hat{H}_{f}$ and $\hat{H}_{I}$ are the free part and the interaction part, respectively. In the rotating wave approximation these quantities take the forms

$$
\begin{aligned}
& \hat{H}_{f}=\omega_{1} \hat{a}_{1}^{\dagger} \hat{a}_{1}+\omega_{2} \hat{a}_{2}^{\dagger} \hat{a}_{2}+\frac{1}{2} \omega_{a} \hat{\sigma}_{z}, \\
& \hat{H}_{I}=g\left(\hat{a}_{1}^{\dagger k_{1}} \hat{a}_{2}^{k_{2}} \hat{\sigma}_{+}+\hat{a}_{2}^{\dagger k_{2}} \hat{a}_{1}^{k_{1}} \hat{\sigma}_{-}\right),
\end{aligned}
$$

where $\hat{\sigma}_{ \pm}$and $\hat{\sigma}_{z}$ are the Pauli spin operators; $\omega_{j},(j=1,2)$ and $\omega_{a}$ are the frequencies of cavity modes $\hat{a}_{j}$ and the atomic transitions, respectively; $g$ is the atom-field coupling constant and $k_{j}$ are the transition parameters, i.e. they represent the number of photons involved in the atomic transition. The derivation of the Hamiltonian (6) from the first principle is given in [30]. Moreover, (6) generalizes various models given in the literature, e.g. [31, 32], and for $k_{1}=k_{2}=1$ it can be interpreted as a cavity version of Raman scattering in which a mode 1 is the pump field, mode 2 is the Stokes field and with no anti-Stokes field [31]. In this case the system represents three-

level atom in $\Lambda$ configuration interacting with the two modes of the field under the assumption of the exact resonance. Applying adiabatic elimination of the second level the effective Hamiltonian reduces to the form of the usual JCM Hamiltonian but the single-mode field operator is replaced by the product of the two modes operators.

We proceed by considering the exact resonance case $\omega_{a}=k_{1} \omega_{1}-k_{2} \omega_{2}$. The dynamical properties of the system can be obtained by means of the unitary operator of the interaction part as

$$
\begin{aligned}
& \hat{U}_{I}(T, 0)=\exp \left(-i \frac{T}{g} \hat{H}_{I}\right) \\
= & \cos (T \hat{D})-i \frac{\sin (T \hat{D})}{g \hat{D}} \hat{H}_{I},
\end{aligned}
$$

where

$$
T=g t, \quad \hat{D}^{2}=\hat{a}_{1}^{\dagger k_{1}} \hat{a}_{1}^{k_{1}} \hat{a}_{2}^{k_{2}} \hat{a}_{2}^{\dagger k_{2}} \hat{\sigma}_{+} \hat{\sigma}_{-}+\hat{a}_{1}^{k_{1}} \hat{a}_{1}^{\dagger k_{1}} \hat{a}_{2}^{\dagger k_{2}} \hat{a}_{2}^{k_{2}} \hat{\sigma}_{-} \hat{\sigma}_{+}
$$


It is worth mentioning that without the rotating wave approximation, i.e. involving the fastly rotating terms in the Hamiltonian, the fully quantum-mechanical system cannot be solved as in (17) since the eigenstates of the Hamiltonian cannot be obtained in a closed form. In this case, numerical techniques such as path-integral approach [33] and perterbative approach [34] have to be used. Inspite of these techniques are very sophisticated the obtained solution will be in an approximate form. Of course, in this case the results of the present paper are no longer valid. For instance, we can mention for the single-mode JCM without RWA the atomic inversion is phase dependent [34].

Additionally, we assume that the two modes are initially prepared in the cat state (41) and the atom is in the superposition of the excited and ground states having the from

$$
|\varphi, \phi\rangle=\cos \varphi|+\rangle+\exp (i \phi) \sin \varphi|-\rangle
$$

where $|+\rangle$ and $|-\rangle$ denote excited and ground atomic states, respectively, and $\phi, \varphi$ are the relative phases between $|-\rangle$ and $|+\rangle$. Therefore the total initial state of the system is

$$
|\Psi(0)\rangle=|\alpha\rangle_{\epsilon_{1}} \bigotimes|\alpha\rangle_{\epsilon_{2}} \bigotimes|\varphi, \phi\rangle
$$

From (17)-(10) the dynamical state of the system is

$$
\begin{aligned}
& |\Psi(T)\rangle=\hat{U}_{I}(T, 0)|\Psi(0)\rangle \\
& =\sum_{n, m=0}^{\infty}\left[F_{1}(n, m, T)\left|+, n, m+k_{2}\right\rangle+F_{2}(n, m, T)\left|-, n+k_{1}, m\right\rangle\right]
\end{aligned}
$$

where the time-dependent coefficients have the forms:

$$
\begin{aligned}
& F_{1}(n, m, T)=C_{n, m+k_{2}} \cos \varphi \cos \left(T \Lambda_{n, m}\right)-i C_{n+k_{1}, m} \exp (i \phi) \sin \varphi \sin \left(T \Lambda_{n, m}\right), \\
& F_{2}(n, m, T)=C_{n+k_{1}, m} \sin \varphi \cos \left(T \Lambda_{n, m}\right) \exp (i \phi)-i C_{n, m+k_{2}} \cos \varphi \sin \left(T \Lambda_{n, m}\right),
\end{aligned}
$$

whereas

$$
\Lambda_{n, m}=\sqrt{\frac{\left(m+k_{2}\right) !\left(n+k_{1}\right) !}{n ! m !}}
$$

and $C_{n, m}=C_{n}^{(1)} C_{m}^{(2)}, C_{n}^{(j)}$ are given by (3). From (11) the entanglement between different components of the system is readily apparent. 
The atomic inversion of the system can be expressed as

$$
\begin{aligned}
& \left\langle\hat{\sigma}_{z}(T)\right\rangle=\sum_{n, m}^{\infty}\left\{\left[C_{n, m+k_{2}}^{2} \cos ^{2} \varphi-C_{n+k_{1}, m}^{2} \sin ^{2} \varphi\right] \cos \left(2 T \Lambda_{n, m}\right)\right. \\
& \left.+\sin \phi \sin (2 \varphi) C_{n, m+k_{2}} C_{n+k_{1}, m} \sin \left(2 T \Lambda_{n, m}\right)\right\} .
\end{aligned}
$$

To calculate the joint phase distribution for the field we find the reduced density operator for the radiation field $\hat{\rho}_{f}(T)$ by tracing (11) over the atomic states as

$$
\begin{aligned}
& \hat{\rho}_{f}(T)=\operatorname{Tr}_{a}[\hat{\rho}(T)] \\
& =\sum_{n, m, n^{\prime}, m^{\prime}}^{\infty}\left[F_{1}(n, m, T) F_{1}^{*}\left(n^{\prime}, m^{\prime}, T\right)\left|n, m+k_{2}\right\rangle\left\langle n^{\prime}, m^{\prime}+k_{2}\right|\right. \\
& \left.+F_{2}(n, m, T) F_{2}^{*}\left(n^{\prime}, m^{\prime}, T\right)\left|n+k_{1}, m\right\rangle\left\langle n^{\prime}+k_{1}, m^{\prime}\right|\right] .
\end{aligned}
$$

In the first line of (15) the notation $\operatorname{Tr}_{a}$ means that we evaluate the trace over the atomic states.

Now we give briefly the relations of the Pegg-Barnett formalism [22], which will be used throughout the paper, however, more details about the technique with various applications can be found in [35]. This formalism is based on introducing a finite $(s+1)$-dimensional space $\Psi$ spanned by the number states $|0\rangle,|1\rangle, \ldots,|s\rangle$. The expectation values of the different hermitian phase operators can be evaluated in the finite dimensional space $\Psi$ and at the final stage the limit $s \rightarrow \infty$ is taken. The hermitian phase operator of the single-mode case is defined as

$$
\hat{\Phi}=\sum_{m=0}^{s} \Theta_{m}\left|\Theta_{m}\right\rangle\left\langle\Theta_{m}\right|,
$$

where the states $\left|\Theta_{m}\right\rangle$ are eigenstates of the phase operator (16) and they form a complete orthonormal basis of $s+1$ states in $\Psi$. Moreover, these states are restricted to lie within a phase window between $\Theta_{0}$ and $2 \pi+\Theta_{0}$. For the system described by the density matrix $\hat{\rho}=\sum_{m, m^{\prime}=0}^{\infty} C_{m} C_{m^{\prime}}^{*}|m\rangle\left\langle m^{\prime}\right|$ the continuum (, i.e. $s$ tends to infinity) phase distribution is defined as

$$
\begin{array}{r}
P(\Theta)=\lim _{s \rightarrow \infty} \frac{s+1}{2 \pi}\left\langle\Theta_{m}|\hat{\rho}| \Theta_{m}\right\rangle \\
=\frac{1}{2 \pi} \sum_{m, m^{\prime}=0}^{\infty} C_{m} C_{m^{\prime}}^{*} \exp \left[i\left(m-m^{\prime}\right) \Theta\right],
\end{array}
$$

where $\Theta_{m}$ has been replaced by the continuous phase variable $\Theta$. Once the phase distribution $P(\Theta)$ is obtained, all the quantum-mechanical phase moments can be calculated as a classical integral 
over $\Theta$ through the relation

$$
\left\langle\hat{\Phi}^{l^{\prime}}\right\rangle=\int_{-\pi}^{\pi} \Theta^{l^{\prime}} P(\Theta, T) d \Theta, \quad l^{\prime}=1,2, \ldots
$$

The phase variance is defined as

$$
\left\langle(\triangle \hat{\Phi})^{2}\right\rangle=\left\langle\hat{\Phi}^{2}\right\rangle-\langle\hat{\Phi}\rangle^{2}
$$

Actually, the generalization of the relations (16)-(19) to the two-mode version is straightforward. In this respect the joint phase distribution associated with the reduced density matrix (15) can be evaluated as

$$
P\left(\Theta_{1}, \Theta_{2}, T\right)=\frac{1}{4 \pi^{2}} \sum_{n, m, n^{\prime}, m^{\prime}}^{\infty} C\left(n, m, n^{\prime}, m^{\prime}, T\right) \exp \left[i\left(n^{\prime}-n\right) \Theta_{1}+i\left(m^{\prime}-m\right) \Theta_{2}\right],
$$

where

$$
\begin{aligned}
& C\left(n, m, n^{\prime}, m^{\prime}, T\right)=F_{1}(n, m, T) F_{1}^{*}\left(n^{\prime}, m^{\prime}, T\right)+F_{2}(n, m, T) F_{2}^{*}\left(n^{\prime}, m^{\prime}, T\right) \\
& =\left[C_{n, m+k_{2}} C_{n^{\prime}, m^{\prime}+k_{2}} \cos ^{2} \varphi+C_{n+k_{1}, m} C_{n^{\prime}+k_{1}, m^{\prime}} \sin ^{2} \varphi\right] \cos \left[T\left(\Lambda_{n, m}-\Lambda_{n^{\prime}, m^{\prime}}\right)\right] .
\end{aligned}
$$

For the sake of simplicity in (21) we restrict the calculation to the case $\phi=0$. Also one can easily prove that

$$
\int_{-\pi}^{\pi} \int_{-\pi}^{\pi} P\left(\Theta_{1}, \Theta_{2}, T\right) d \Theta_{1} d \Theta_{2}=1
$$

The single-mode phase distribution of the $j$ th mode can be obtained from (20) via the relation

$$
\int_{-\pi}^{\pi} P\left(\Theta_{j^{\prime}}, \Theta_{j}, T\right) d \Theta_{j^{\prime}}=P\left(\Theta_{j}, T\right), \quad j \neq j^{\prime}
$$

It is worth mentioning that (20) can be expressed in terms of the single-mode phase distribution as

$$
\begin{array}{r}
P\left(\Theta_{1}, \Theta_{2}, T\right)=\frac{1}{4 \pi^{2}}\left\{2 \pi\left[P\left(\Theta_{1}, T\right)+P\left(\Theta_{2}, T\right)\right]-1\right. \\
+2 \sum_{n>n^{\prime}}^{\infty} \sum_{m>m^{\prime}}^{\infty}\left[C_{+}\left(n, m, n^{\prime}, m^{\prime}, T\right) \cos \left[\left(n-n^{\prime}\right) \Theta_{1}\right] \cos \left[\left(m-m^{\prime}\right) \Theta_{2}\right]\right. \\
\left.\left.+C_{-}\left(n, m, n^{\prime}, m^{\prime}, T\right) \sin \left[\left(n-n^{\prime}\right) \Theta_{1}\right] \sin \left[\left(m-m^{\prime}\right) \Theta_{2}\right]\right]\right\},
\end{array}
$$

where

$$
C_{ \pm}\left(n, m, n^{\prime}, m^{\prime}, T\right)=C\left(n^{\prime}, m, n, m^{\prime}, T\right) \pm C\left(n, m, n^{\prime}, m^{\prime}, T\right)
$$


Expression (24) is relevant for numerical tasks.

To investigate the fluctuation in the phase distribution for the system under consideration we have to evaluate different moments for the phase operators, which give

$$
\begin{aligned}
& \left\langle\hat{\Phi}_{1}\right\rangle=0 \\
& \left\langle\hat{\Phi}_{1}^{2}\right\rangle=\frac{\pi^{2}}{3}+2 \sum_{n \neq n^{\prime}, m}^{\infty} C\left(n, m, n^{\prime}, m, T\right) \frac{(-1)^{n^{\prime}-n}}{\left(n^{\prime}-n\right)^{2}}, \\
& \left\langle\hat{\Phi}_{1} \hat{\Phi}_{2}\right\rangle=-\sum_{n \neq n^{\prime}, m \neq m^{\prime}}^{\infty} C\left(n, m, n^{\prime}, m^{\prime}, T\right) \frac{(-1)^{n^{\prime}+m^{\prime}-m-n}}{\left(m^{\prime}-m\right)\left(n^{\prime}-n\right)} .
\end{aligned}
$$

The sum- and difference-phase operators are defined as

$$
\hat{\Phi}_{ \pm}=\hat{\Phi}_{1} \pm \hat{\Phi}_{2}
$$

where "+" and "-" denote sum-phase and difference-phase operators, respectively. Therefore, the sum- and difference-phase variances can be expressed in the following formula

$$
\begin{aligned}
& \left\langle\left(\triangle \hat{\Phi}_{ \pm}\right)^{2}\right\rangle=\left\langle\left(\triangle \hat{\Phi}_{1}\right)^{2}\right\rangle+\left\langle\left(\triangle \hat{\Phi}_{2}\right)^{2}\right\rangle \pm h_{1,2} \\
& h_{1,2}=2\left[\left\langle\hat{\Phi}_{1} \hat{\Phi}_{2}\right\rangle-\left\langle\hat{\Phi}_{1}\right\rangle\left\langle\hat{\Phi}_{2}\right\rangle\right] .
\end{aligned}
$$

Moreover, as the mean-photon number and the phase are conjugate quantities. It is reasonable to investigate the fluctuation in the mean-photon number for the single-mode and compound-mode cases $\left\langle\left(\triangle \hat{n}_{j}\right)^{2}\right\rangle$ and $\left\langle\left(\triangle \hat{n}_{ \pm}\right)^{2}\right\rangle$, where $\hat{n}_{j}=\hat{a}_{j}^{\dagger} \hat{a}_{j}$ and $\hat{n}_{ \pm}=\hat{n}_{1} \pm \hat{n}_{2}$.

On the other hand, we address the relationship between the behaviors of the phase distribution and the $W$ function at the phase space origin for the system under consideration. The reasons for this will be clear shortly. The joint Wigner function can be easily evaluated using a technique similar to that given in [21] as

$$
\begin{aligned}
& W\left(\chi_{1}, \chi_{2}, T\right)=\frac{\exp \left(-\left|\chi_{1}\right|^{2}-\left|\chi_{2}\right|^{2}\right)}{\pi^{2}} \sum_{n, m, n^{\prime}, m^{\prime}}^{\infty}(-1)^{n^{\prime}+m^{\prime}} \chi_{1}^{n-n^{\prime}} \chi_{2}^{m-m^{\prime}} 2^{\frac{n+m-n^{\prime}-m^{\prime}}{2}} \\
& \times\left[(-1)^{k_{2}} F_{1}(n, m, T) F_{1}^{*}\left(n^{\prime}, m^{\prime}, T\right) \sqrt{\frac{n^{\prime} !\left(m^{\prime}+k_{2}\right) !}{n !\left(m+k_{2}\right) !}} \mathrm{L}_{n^{\prime}}^{n-n^{\prime}}\left(2\left|\chi_{1}\right|^{2}\right) \mathrm{L}_{m^{\prime}+k_{2}}^{m-m^{\prime}}\left(2\left|\chi_{2}\right|^{2}\right)\right. \\
& \left.+(-1)^{k_{1}} F_{2}(n, m, T) F_{2}^{*}\left(n^{\prime}, m^{\prime}, T\right) \sqrt{\frac{\left(n^{\prime}+k_{1}\right) ! m^{\prime} !}{\left(n+k_{1}\right) ! m !}} \mathrm{L}_{n^{\prime}+k_{1}}^{n-n^{\prime}}\left(2\left|\chi_{1}\right|^{2}\right) \mathrm{L}_{m^{\prime}}^{m-m^{\prime}}\left(2\left|\chi_{2}\right|^{2}\right)\right]
\end{aligned}
$$

where $\chi_{j}=x_{j}+i y_{j}, j=1,2$ and $\mathrm{L}_{n}^{\nu}($.$) are associated Laguerre polynomials of order n$. The $W$ function of the single-mode case can be obtained from (29) by tracing over the other mode. Now 

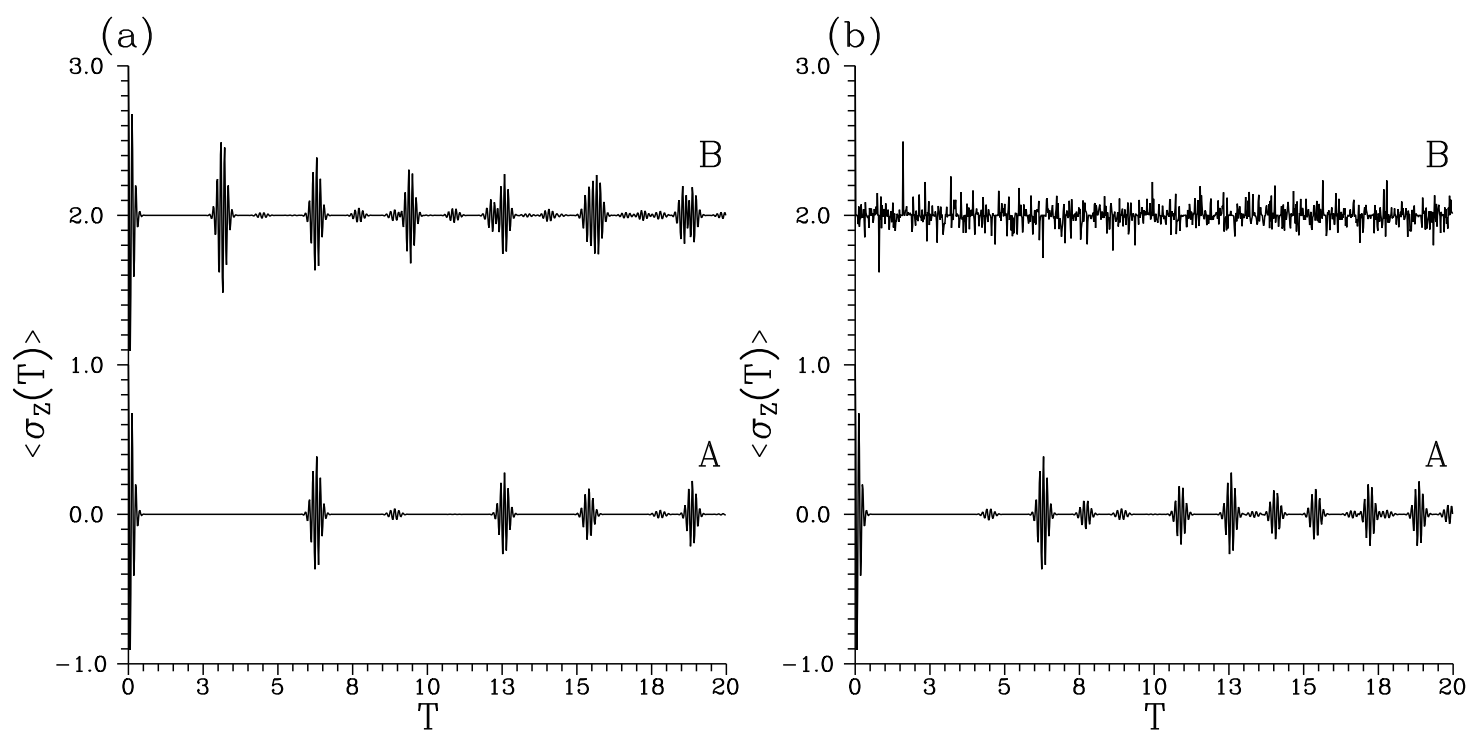

FIG. 1: The atomic inversion $\left\langle\hat{\sigma}_{z}(T)\right\rangle$ versus the scaled time $T$ for $\varphi=0$ and the modes are prepared initially in different types of the initial states with $\left|\alpha_{j}\right|=5$. (a) $\left(\epsilon_{1}, \epsilon_{2}, k_{1}, k_{2}\right)=(0,0,1,1)$ (curve A), $(1,1,1,1)$ (curve B); (b) $\left(\epsilon_{1}, \epsilon_{2}, k_{1}, k_{2}\right)=(1,0,1,1)$ (curve A), $(1,1,2,2)$ (curve B). The curves B are given for $\left\langle\hat{\sigma}_{z}(T)\right\rangle+2$.

the $W$ functions associated with the first mode $\left(W_{1}\right)$, second mode $\left(W_{2}\right)$ and compound modes $(W)$ at the phase space origin, i.e. $\chi_{j}=0$, can be expressed as

$$
\begin{gathered}
W_{1}(0, T)=\frac{1}{\pi} \sum_{n, m=0}^{\infty}(-1)^{n}\left[\left|F_{1}(n, m, T)\right|^{2}+(-1)^{k_{1}}\left|F_{2}(n, m, T)\right|^{2}\right], \\
W_{2}(0, T)=\frac{1}{\pi} \sum_{n, m=0}^{\infty}(-1)^{m}\left[(-1)^{k_{2}}\left|F_{1}(n, m, T)\right|^{2}+\left|F_{2}(n, m, T)\right|^{2}\right], \\
W(0, T)=\frac{1}{\pi^{2}} \sum_{n, m=0}^{\infty}(-1)^{m+n}\left[(-1)^{k_{2}}\left|F_{1}(n, m, T)\right|^{2}+(-1)^{k_{1}}\left|F_{2}(n, m, T)\right|^{2}\right] .
\end{gathered}
$$

Finally, as it is obvious that the system includes various parameters, which make the analysis to be difficult. Thus we restrict ourselves to the parameters, which can give significant results. Also in the text the statement standard JCM means $k_{1}=0, k_{2}=1$, the optical cavity field and atom are initially prepared in coherent state and in the atomic excited state, respectively. 


\section{RESULTS AND DISCUSSIONS}

In this section we investigate the quantities, which have been calculated in section 2. Such investigation includes the atomic inversion, $W$ function, phase distribution and and the phase and mean-photon variances.

\section{A. Atomic inversion and Wigner function}

As it is well known for the standard JCM that there is a direct connection between the phase distribution and $\left\langle\hat{\sigma}_{z}(T)\right\rangle$ [23]. Additionally, quite recently it has been proved that there is a relation between $\left\langle\hat{\sigma}_{z}(T)\right\rangle$ and the evolution of the $W$ function at the phase space origin [21]. This relationship is supported by the recent developments in photon counting experiment [36] and trapped ion technique [37, 38], where the measurements have been focused on the origin of the $W$ function in the phase space. The natural question is: what would be the situation for the TMJCM? In this part we answer this question by investigating the behavior of the $\left\langle\hat{\sigma}_{z}(T)\right\rangle$ and showing its connection with the $W$ function for the system under consideration.

Firstly one of the most interesting phenomenon, which is representative to the single-mode JCM, is the RCP in the evolution of the $\left\langle\hat{\sigma}_{z}(T)\right\rangle$. Much attention has been focused on this phenomenon because it provides evidence of the granularity of the radiation field. It is worth mentioning that the observation of RCP has been performed via one-atom mazer [13]. Moreover, schemes for measuring RCP via homodyne detection [39], photon counting experiment and homodyne tomography [40] have been reported, too. For more details related to such phenomenon reader can consult, e.g. [2]. On the other hand, RCP associated with the TMJCM is very complicated compared to that one associated with the standard JCM where for the TMJCM the positions of the revivals in the time domain are independent of the intensities of the initial modes provided that $\left\langle\hat{n}_{j}(0)\right\rangle=\bar{n}_{j}>>1$. Such behavior has been explained partially in [31]. Also in [32] it has been shown that there are secondary revivals coming from the complicated interferences, which are resulting from the double summations in the atomic inversion (cf. (14)). We should stress that the origin of the RCP in the $\left\langle\hat{\sigma}_{z}(T)\right\rangle$ of the TMJCM is the strong entanglement between the bosonic and fermonic systems. To be more specific, restricting the discussion to the case $k_{1}=k_{2}=1$, when the atom is classically treated the Hamiltonian (5) describes the up conversion process [41, 42], which is representative well by switching the energy between the signal and idler modes. Conversely, when the field is classically treated, i.e. $\hat{a}_{j} \rightarrow\left|\alpha_{j}\right| \exp \left(-i \bar{\phi}_{j}\right)$, (5) reduces to two-level atom interacting with the 

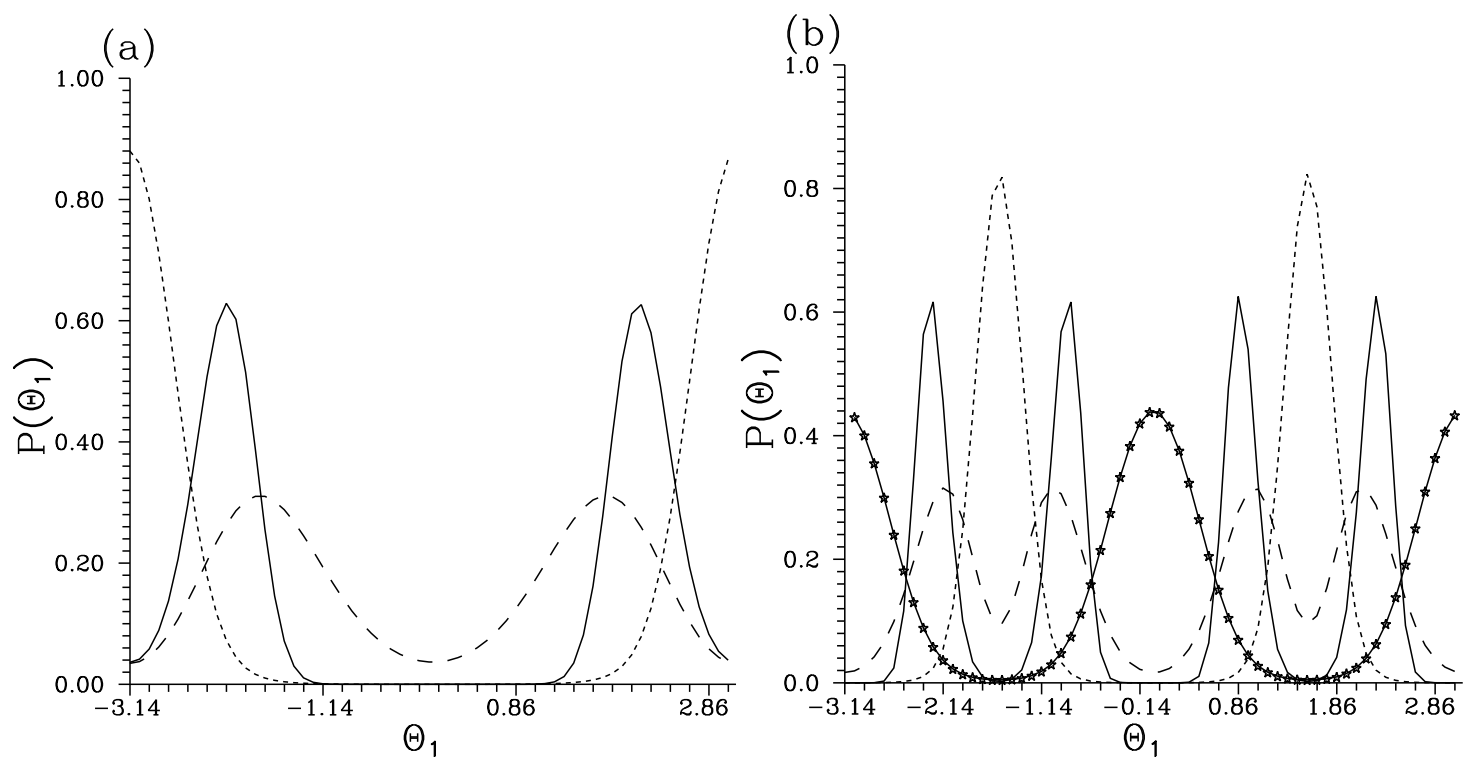

FIG. 2: The phase distribution $P\left(\Theta_{1}\right)$ of the first mode when $\left|\alpha_{j}\right|=5, \varphi=0, \phi=0, k_{1}=k_{2}=1$ and for $\left(\epsilon_{1}, \epsilon_{2}\right)=(0,0)(a)$ and $(1,1)(b)$. For (a) $T=4.42$ (solid curve-collapse time), 6.2999 (short-dashed curverevival time) and 9.32 (long-dashed curve-secondary revival time). For (b) $T=1.8$ (solid curve-collapse time), 3.22 (short-dashed curve-revival time) and 4.4 (long-dashed curve-secondary revival time). In (b) the star-centered curve is given for $\left(\epsilon_{1}, \epsilon_{2}\right)=(0,1)$ and $T=6.3$ (revival time).

classical fields. In this case $\left\langle\hat{\sigma}_{z}(T)\right\rangle$ exhibits steady-state Rabi frequency. In Figs. 1(a) and (b) we have plotted $\left\langle\hat{\sigma}_{z}(T)\right\rangle$ against the scaled time $T$ when the atom is in the excited state and the modes are initially prepared in different types of states as indicated. In Fig. 1(a) for curve A one can observe the revivals and secondary revivals representative to TMJCM. For curve B, where the interference in phase space starts to play a role, the revivals and secondary revivals are more pronounced than those shown in curve $\mathrm{A}$ and the behavior becomes more systematic, i.e. each revival is followed by a secondary revival. This can be explained as follows. For curve B the density matrix (4) has two parts, which are $\hat{\rho}_{M}$ and $\hat{\rho}_{I}$ (cf. (44)). Each of which provides its own RCP pattern and consequently they interfere with each other showing such behavior. Furthermore, it seems that the revival time for the curve $A$ is two times greater than that for curve $B$. This can be partially explained for $\bar{n}_{j}>>1$ and $k_{1}=k_{2}=1$. In this case the photon distribution for both initial coherent state and even coherent state possess Poissonian envelope. This means that the terms contributing effectively to the summations in (14) are those for which the pairs $\left(\alpha_{1}, \alpha_{2}\right)$ and $(n, m)$ are comparable. Now we assume that the arguments of $\cos ($.$) for initial coherent light is \lambda_{1}$ with interaction time $T$ and for initial even coherent light is $\lambda_{2}$ with interaction time $T^{\prime}$, i.e.

$$
\lambda_{1}=2 T \sqrt{n(m+1)}, \lambda_{2}=2 T^{\prime} \sqrt{2 n(2 m+1)} .
$$




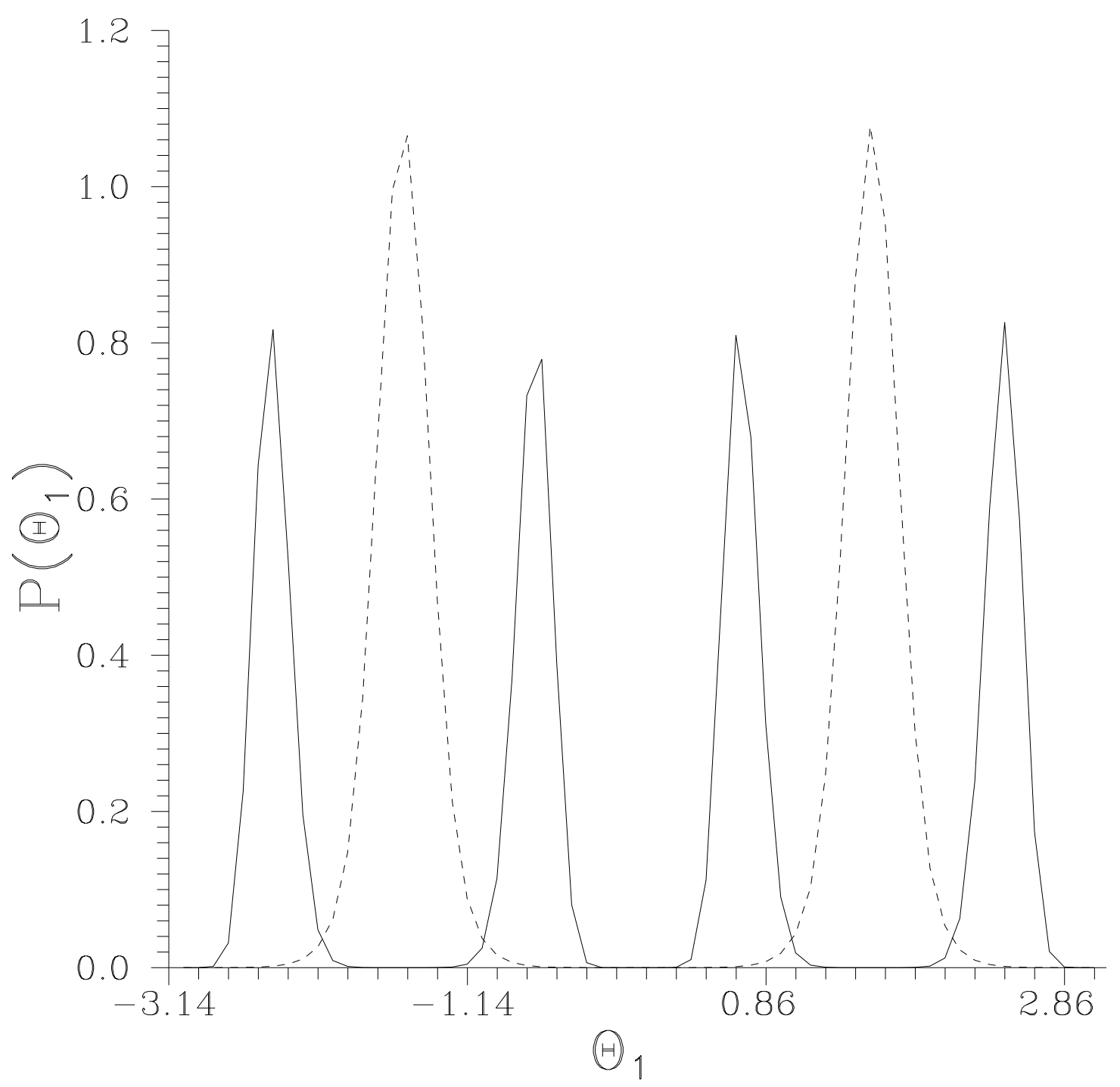

FIG. 3: The phase distribution $P\left(\Theta_{1}\right)$ of the single-mode $\mathrm{JCM}$, i.e. $k_{1}=0, k_{2}=1$, when $\left|\alpha_{1}\right|=5, \varphi=$ $0, \phi=0, \epsilon_{1}=1$ and for $T=7$ (solid curve-collapse time) and 16.4 (dashed curve- revival time).

The revivals in $\left\langle\hat{\sigma}_{z}(T)\right\rangle$ for both cases occur when $\lambda_{1} \simeq 2 m^{\prime} \pi, \quad \lambda_{2} \simeq 2 m^{\prime} \pi$, where $m^{\prime}$ is a positive integer. In the strong-intensity regime $\lambda_{1}$ and $\lambda_{2}$ can be simplified as

$$
\lambda_{1}=2 T \sqrt{\bar{n} \bar{m}}, \lambda_{2}=4 T^{\prime} \sqrt{\bar{n} \bar{m}}
$$

Therefore, the relation between the revival times of the two cases is $T_{R}^{\prime}=\frac{T_{R}}{2}$, where the subscript $R$ indicates that this time is associated with the revival pattern. On the other hand, in Fig. 1(b) the curve $A$ is given for $k_{j}=1$ when one of the modes is initially in coherent states and the other is in even coherent states; while the curve B is given for $k_{j}=2$ and the modes are initially in even coherent states. The secondary revivals in curve A are more pronounced than those in Fig. 1(a). Nevertheless, the curve B, which is given for higher-order photon transition, exhibits 
chaotic behavior resulting from the entanglement between different modes. This can be realised by comparing this behavior with that of the atomic inversion of the two-photon-single-mode JCM [21], which exhibits compact and systematic revivals. From the above discussion we can conclude that the occurrence of the RCP and the secondary revivals in $\left\langle\hat{\sigma}_{z}(T)\right\rangle$ for the TMJCM basically depends on the values of the transition parameters $k_{j}$ and on the type of the initial distribution of the modes. Nevertheless, it is insensitive to the values of the initial intensities of the modes.

Now we draw the attention to the behavior of the $W$ function, in particular, the evolution of the $W$ function at the phase space origin. We start the discussion by assuming that the two modes are initially prepared in even coherent states and $k_{1}=k_{2}=$ odd number. In this case (30)-(32) reduce to

$$
W_{1}(0, T)=\frac{1}{\pi}\left\langle\hat{\sigma}_{z}(T)\right\rangle, \quad W_{2}(0, T)=\frac{-1}{\pi}\left\langle\hat{\sigma}_{z}(T)\right\rangle, \quad W(0, T)=\frac{-1}{\pi^{2}} .
$$

Expressions (35) provide an important fact: The evolution of the phase-space origin of the singlemode $W$ function can carry information on the atomic inversion of the system. However, the joint $W$ function is localized in phase space giving maximum nonclassicality, i.e. maximum negativity. On the other hand, when the number of photons involved in the atomic transition is even, i.e. $k_{1}=k_{2}=$ even number, the $W$ functions of the single mode and compound modes are localized in phase space:

$$
W_{1}(0, T)=W_{2}(0, T)=\frac{1}{\pi}, \quad W(0, T)=\frac{1}{\pi^{2}}
$$

Moreover, when $k_{1}$ is odd, say, and $k_{2}$ is even we obtain

$$
W(0, T)=\frac{1}{\pi} W_{1}(0, T)=\frac{1}{\pi^{2}}\left\langle\hat{\sigma}_{z}(T)\right\rangle, \quad W_{2}(0, T)=\frac{1}{\pi} .
$$

Finally, when $k_{1}, k_{2}$ are odd and the two modes are initially prepared in a general quantum states, e.g. coherent states-restricting the discussion to the excited atomic state-(30) and (32) take the forms

$$
\begin{gathered}
W_{1}(0, T)=\frac{1}{\pi} \sum_{n, m=0}^{\infty} C_{n, m+k_{2}}^{2} \cos \left(2 T \Lambda_{n, m}+n \pi\right), \\
W(0, T)=-W_{1}(0,0) W_{2}(0,0),
\end{gathered}
$$

where $W_{j}(0,0)$ is the initial value of the $W$ function of the $j$ th mode at the phase space origin. Expression (38)-apart from the prefactor $1 / \pi$-is typical to that of the $\left\langle\hat{\sigma}_{z}(T)\right\rangle$ but with additional factor, i.e. $(-1)^{n}$, however, the joint $W$ function is localized in the phase space. Actually, we have 

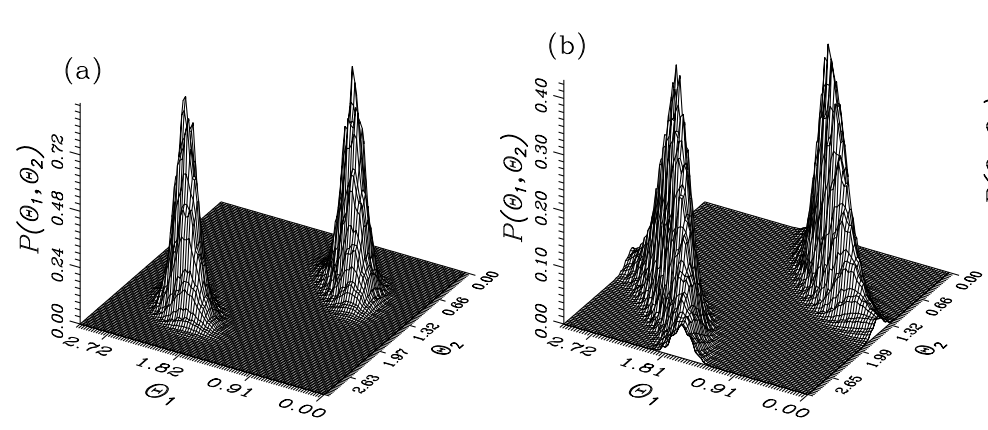

(c)

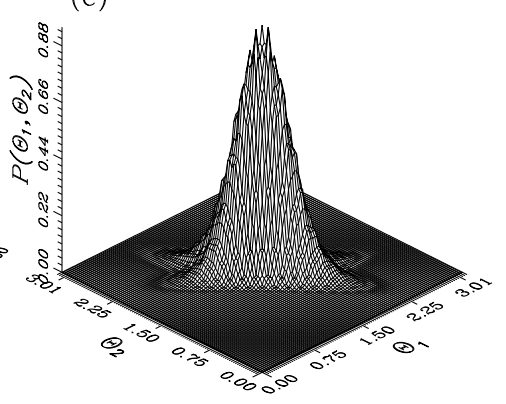

FIG. 4: The joint phase distribution $P\left(\Theta_{1}, \Theta_{2}\right)$ for $\left|\alpha_{j}\right|=5, \varphi=0, \phi=0, k_{1}=k_{2}=1,\left(\epsilon_{1}, \epsilon_{2}\right)=(1,1)$ and for $T=1.8(\mathrm{a}), 4.4(\mathrm{~b})$ and $3.22(\mathrm{c})$.

numerically checked the behavior of (38) and found that it can provide typical curves to those of the corresponding atomic inversion. Therefore, for specific values of the interaction parameters the evolution of the $W$ functions at the phase space origin represents the atomic inversion of the system, in particular, when $k_{1}$ or $k_{2}$ (or both of them) is an odd number. This leads to the fact that for TMJCM there is a connection between the behavior of both the atomic inversion, the $W$ function and the phase space distribution of the single mode as well as the compound modes, as we shall show shortly. This situation is similar to that of the standard JCM [23].

\section{B. Phase distribution}

For the standard JCM it has been shown that there is a relationship between the time evolution of the atomic inversion, the phase distribution and $Q$ function. For instance, the RCP is reflected in the behavior of the phase distribution of the radiation field [23] where the single-peak structure of the initial phase distribution splits into two peaks, which are rotated in opposite directions. At this moment $\left\langle\hat{\sigma}_{z}(T)\right\rangle$ exhibits collapse pattern. Furthermore, as the interaction proceeds the two peaks of the distribution are collided showing single-peak form, however, $\left\langle\hat{\sigma}_{z}(T)\right\rangle$ shows revivals. Here we show that similar behavior can occur for the single-mode and compound-mode cases of the TMJCM. Additionally, we investigate the influence of the higher-order photon transition $k_{j}>1$ on such behavior. It is worth reminding that the behavior of the $\left\langle\hat{\sigma}_{z}(T)\right\rangle$ of the TMJCM is rather complicated compared to that of the standard JCM (see Figs. 1). Actually, we found that the behavior of the first and second modes phase distribution are typical so that the discussion would be restricted to that of the first mode only. Furthermore, as it is difficult to treat the behavior of the phase distributions analytically we use the numerical technique (see Figs. 2-5). Figs. 2(a) and (b) are given for the single-mode phase distribution when the two modes are initially prepared in 


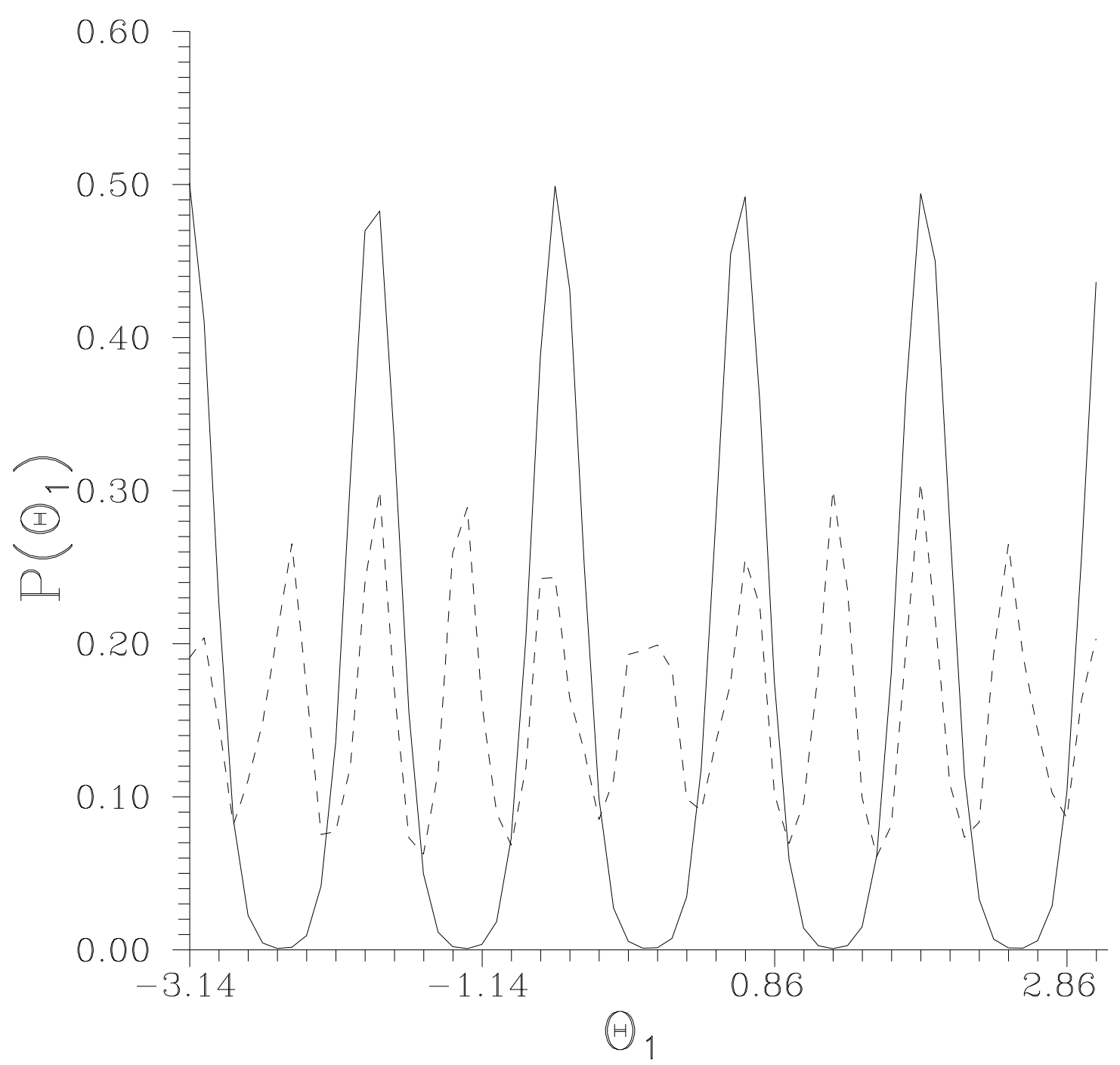

FIG. 5: The phase distribution $P\left(\Theta_{1}\right)$ of the first mode for $T=5,\left|\alpha_{j}\right|=5, \varphi=0, \phi=0, k_{1}=k_{2}=2$ and for $\left(\epsilon_{1}, \epsilon_{2}\right)=(0,0)$ (solid curve) and $(1,1)$ (dashed curve).

coherent and even coherent states, respectively, for collapse time (solid curve), secondary revival time (long-dashed curve) and revival time (short-dashed curve). In Fig. 2(b) the star-centered curve is given for the case $\left(\epsilon_{1}, \epsilon_{2}\right)=(0,1)$ and the corresponding revival time $T=6.3$. In Fig. 2(a) one can see that $P\left(\Theta_{1}\right)$ exhibits two-peak structure for both collapse time (with two maxima around $\Theta_{1} \simeq \pm 2 \pi / 3$ ) and secondary-revival time (with two maxima around $\Theta_{1} \simeq \pm \pi / 2$ ), however, those of the latter are border and shorter than those of the former. In fact such behavior (, i.e., the occurrence of the two-peak structure in the phase distribution) is quite similar to that of the even (odd) coherent states [43]. Furthermore, this behavior is representative to the standard JCM indicating generation of the cat states. Nevertheless, it is difficult to consider such conclusion here owing to the strong entanglement between different components of the system. For the revival time 
$P\left(\Theta_{1}\right)$ exhibits two wings around $\Theta_{1} \rightarrow \pm \pi$ (see short-dashed curve), i.e. it is incomplete peak. In Fig. 2(b), one can see that four-peak structure occurring through the collapse and secondary revival times for the initial even coherent states, which are combined at the revival time providing two-peak structure. Interestingly, when one of the modes is initially prepared in coherent state and the other is in even coherent state we have seen that for collapse and secondary revival times the behavior is typical to that of the two modes, which are initially prepared in the cat states (see solid and long-dashed curves in Fig. 2(b)), however, for the revival time we obtain single-peak around $\Theta_{1} \simeq 0$ and two wings as $\Theta_{1} \rightarrow \pm \pi$ (see the star-centered curve in Fig. 2(b)). This indicates that the influence of the interference in phase space on the behavior of the phase distribution is more pronounced in the course of the revival time. Nevertheless, the influence of the entanglement on the behavior of the distribution can be realised by comparing Fig. 3, which is given for the single-mode JCM when the mode is initially prepared in the cat state, with the Fig. 2(b), in particular, the solid and short-dashed curves in the two figures. Such comparison shows that there is agreement and disagreement in the phase distribution of the two systems. For instance, the two systems exhibit four (two) peaks in the course of the collapse (revival) time, however, those of the single-mode JCM are narrower and higher than those of the TMJCM. The reason for occurring the same number of peaks in the distribution of the two systems is the term $\cos \left(\left(n-n^{\prime}\right) \Theta_{1}\right)$. Also from (35) and (38) Figs. 2 can provide information on the evolution of the $W$ function at the phase space origin.

Now we turn the attention to the joint phase distribution. We have noted that the evolution of the $P\left(\Theta_{1}, \Theta_{2}, T\right)$ reflects well the behavior of the $\left\langle\hat{\sigma}_{z}(T)\right\rangle$ (see Figs. 4(a)-(c) for given values of the parameters, which are the same as those of Fig. 2b). Based on the fact $P\left(\Theta_{1}, \Theta_{2}, T\right)$ is symmetric in $\Theta_{1}$ and $\Theta_{2}$ we have plotted Figs. 4 only over the region $0 \leq \Theta_{j} \leq \pi$. Generally, there is a similarity between the single-mode and compound-mode phase distribution. To be more specific, for initial even coherent light, $P\left(\Theta_{1}, \Theta_{2}, T\right)$ exhibits two peaks over the specified region in the course of the collapse and secondary revival times, which reduce to single peak (recombination of the peaks) through the revival time. Moreover, the peaks, which are related to the secondary revival time, are broader than those of the collapse time (compare Fig. 4(a) and Fig. 4(b)). Also the influence of the interference in phase space on the behavior of the joint phase distribution is remarkable as multipeak structure.

We conclude this part by shedding the light on the two facts. (i) For $k_{j}>1$ where the atomic inversion exhibiting chaotic behavior the phase distributions show multipeak structure, which is insensitive to the values of the interaction time (, i.e. steady state phase distribution). Of course 

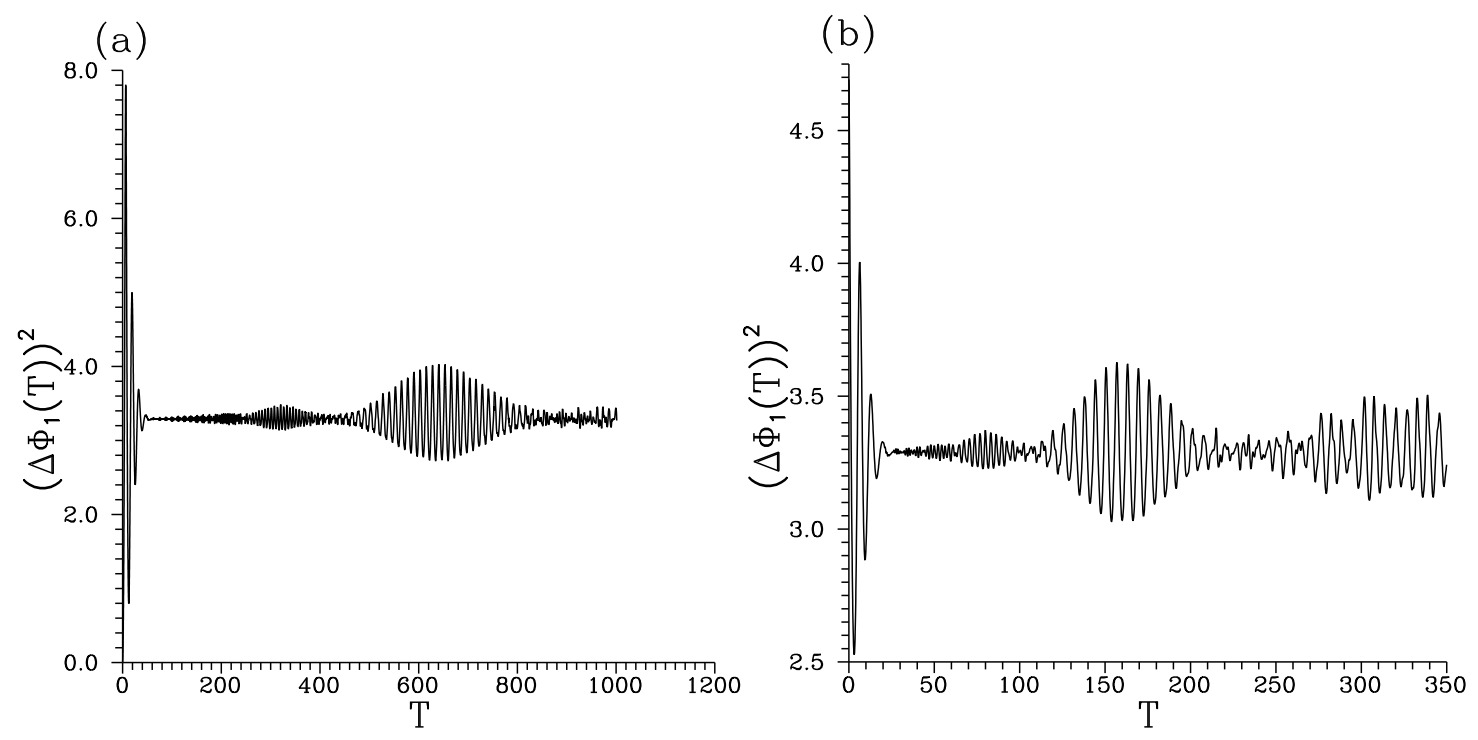

FIG. 6: The single-mode phase variance $\left\langle\left(\triangle \hat{\Phi}_{1}\right)^{2}\right\rangle$ of the first mode versus the scaled time $T$ for $\left|\alpha_{j}\right|=$ $5, \varphi=0, \phi=0, k_{1}=k_{2}=1$, and for $\left(\epsilon_{1}, \epsilon_{2}\right)=(0,0)$ (a) and $(1,1)$ (b).

the number of peaks for the initial cat states are approximately two times greater than those for the initial coherent light (see Fig. 5 for given values of the parameters). (ii) As it is well known that for the standard JCM "coherent trapping" (, i.e. $\left\langle\hat{\sigma}_{z}(T)\right\rangle \simeq 0$ ) can occur [44]. Similarly this can occur for the TMJCM under certain condition (cf. (14)). Nevertheless, this cannot be remarked in the behavior of the phase distribution as a result of the fact that the phase distribution is normalized (cf. (22)). Therefore, the relationship between the behavior of $\left\langle\hat{\sigma}_{z}(T)\right\rangle, P\left(\Theta_{j}, T\right)$ and $P\left(\Theta_{1}, \Theta_{2}, T\right)$ occurs only when the two-level atom is either in the excited or ground state. Thus we have limited the discussion to these cases. Finally, we have found also that the behavior of $P\left(\Theta_{j}, T\right)$ and $P\left(\Theta_{1}, \Theta_{2}, T\right)$ are independent of the type of the initial cat states and the initial atomic states.

\section{Phase fluctuations}

For the standard JCM it has been shown that the phase variance exhibits collapses and revivals about the long-time interaction, and there appear main revivals and secondary revivals with different periods [24]. The origin of this phenomenon is in the phase correlations between the different eigenstates of the photon number that make contributions to the orientated distribution of phase, and the contribution due to the correlations between the neighboring Fock states is much larger than the others [24]. Actually, we have seen that this phenomenon can occur for the TMJCM for 
interaction time shorter than that of the single-mode JCM (see Figs. 6-7 for given values of the interaction parameters). From Fig. 6(a) we observe that the RCP as well as secondary revival occur around $\pi^{2} / 3$, around the random phase distribution. Comparison of this figure with the Fig. 1 in [24] shows that the entanglement between the two modes in the interaction with the twolevel atom makes the RCP in the phase variances occurring through interaction time several times smaller than that of the standard JCM. This behavior can be roughly explained in the following sense. We restrict the analysis to the case $k_{1}=k_{1}=1$ when the two modes are initially prepared in the coherent states with strong intensities (, i.e. $\left|\alpha_{j}\right|>>1, \quad j=1,2$ ) and the atom is in the excited state. From (26) the phase variance of the first mode $\left\langle\left(\triangle \hat{\Phi}_{1}\right)^{2}\right\rangle$ takes the form

$$
\begin{aligned}
& \left\langle\left(\triangle \hat{\Phi}_{1}\right)^{2}\right\rangle=\frac{\pi^{2}}{3}+4 \sum_{n>n^{\prime}}^{\infty} \sum_{m=0}^{\infty} C_{n}^{(1)} C_{n^{\prime}}^{(1)}\left(C_{m+1}^{(2)}\right)^{2} \frac{(-1)^{n^{\prime}-n}}{\left(n^{\prime}-n\right)^{2}} \\
& \times \cos \left[T \sqrt{m+1}\left(\sqrt{n+1}-\sqrt{n^{\prime}+1}\right)\right] .
\end{aligned}
$$

It is worth mentioning that the expression of the phase variance of the standard JCM can be obtained from (40) by dropping the summation $\sum_{m=0}^{\infty}$ and the notations related to the second mode. In this case the argument of the $\cos ($.$) , i.e. the argument of the dynamical term of the$ standard JCM, is

$$
T Z_{n, n^{\prime}}=T\left(\sqrt{n+1}-\sqrt{n^{\prime}+1}\right)
$$

Therefore, the phase variance can exhibit revivals, i.e. $\cos ($.$) provides maxima, only when$

$$
T Z_{n, n^{\prime}}=l \pi, \quad l \text { is integer }
$$

where $T$ is the revival time, say, of the phase variance of the standard JCM. Now we draw the attention to the $\left\langle\left(\triangle \hat{\Phi}_{1}\right)^{2}\right\rangle$ of the TMJCM given by (40). In the strong-intensity regime, i.e. $\sqrt{\bar{m}}=$ $\left|\alpha_{2}\right|>>1$, we can apply the harmonic approximation technique [21] to evaluate the summation related to the second mode where $\left(C_{m}^{(2)}\right)^{2}$ has Poissonian distribution. In this case (40) reduces to

$$
\begin{aligned}
& \left\langle\left(\triangle \hat{\Phi}_{1}\right)^{2}\right\rangle=\frac{\pi^{2}}{3}+4 \sum_{n>n^{\prime}}^{\infty} C_{n}^{(1)} C_{n^{\prime}}^{(1)} \frac{(-1)^{n^{\prime}-n}}{\left(n^{\prime}-n\right)^{2}} \\
& \times \exp \left[-2 \bar{m} \sin ^{2}\left(\frac{T Z_{n, n^{\prime}}}{4 \sqrt{\bar{m}}}\right)\right] \cos \left[\frac{1}{2} T \sqrt{\bar{m}} Z_{n, n^{\prime}}+\bar{m} \sin \left(\frac{T Z_{n, n^{\prime}}}{4 \sqrt{\bar{m}}}\right)\right] .
\end{aligned}
$$

Expression (43) can provide revivals when the exponential term is maximum, i.e.

$$
\frac{T^{\prime} Z_{n, n^{\prime}}}{4 \sqrt{m}}=l \pi
$$


where $T^{\prime}$ denotes the revival time of the $\left\langle\left(\triangle \hat{\Phi}_{1}\right)^{2}\right\rangle$ of the TMJCM. From (42) and (44) we arrive at

$$
\frac{T^{\prime}}{4 \sqrt{\bar{m}}}=T
$$

Expression (45) shows that $T^{\prime}<T$. In other words, the RCP in $\left\langle\left(\triangle \hat{\Phi}_{1}\right)^{2}\right\rangle$ for the TMJCM occurs for interaction time $4 \sqrt{\bar{m}}$ times shorter than that of the standard JCM. We proceed that the RCP in $\left\langle\left(\triangle \hat{\Phi}_{1}\right)^{2}\right\rangle$ can occur through interaction time smaller than that shown in Fig. 6(a) by preparing the two modes initially in the cat states. This is shown in Fig. 6(b) and can be proved "roughly" using procedures as those given above. Moreover, the explanation used for the behavior of the atomic inversion of this case can be adopted here.

On the other hand, for the evolution of the phase variances of the standard JCM there are two important cases, which are three- and four-photon transition, i.e. $k_{1}=0, k_{2}=3$ and 4 . The phase variances for these cases exhibit significant RCP, in particular, the four-photon transition case whose phase variance exhibits periodic revivals with period $\pi$. Moreover, these revivals almost restore their initial amplitudes and widths, and they are independent of the intensities of the initial modes [24]. Surprisingly, we have noted-apart from the amplitudes of the revivals-that there is a one-to-one correspondence between the behavior of the phase variance of the standard JCM and that of the TMJCM. In other words, we have seen that the behavior of the single-mode phase variances of the cases $k_{1}+k_{2}=3$ (provided that $k_{j} \neq 0, \quad j=1,2$ ) and $k_{1}=k_{2}=2$ associated with the TMJCM are similar to those of the standard JCM. This information can be realised from Figs. 7(a) and (b). From Fig. 7(a) we can see a systematic behavior, i.e. each revival is followed by secondary revival and the initial revivals are restored. Furthermore, the revivals and secondary revivals are intensities independent (provided that $\left|\alpha_{j}\right|>>1$ ) and they occur periodically with period $\pi / 2$, i.e. half of that of the standard JCM. The origin of such behavior is in the twophoton transition and in the interference in phase space (compare Fig. 7(a) with Fig. 4 in [24]). Furthermore, the comparison of Fig. 7(b) with Fig. 3 in [24] is instructive, where one can realise the role of the entanglement and the interference in phase space.

Generally, the occurrence of the RCP in the $\left\langle\left(\triangle \hat{\Phi}_{1}\right)^{2}\right\rangle$ cannot occur in the $\left\langle\left(\triangle \hat{\Phi}_{ \pm}\right)^{2}\right\rangle$ where $h_{1,2} \neq 0$ (cf. (26) ). We shed the light on the behavior of the mean-photon number variances. It is worth reminding that the mean-photon numbers and the phase operators are conjugate quantities (noncommuting operators). In Figs. 8(a), (b) and (c) we plot $\left.\left.\left\langle\left(\triangle \hat{n}_{1}\right)^{2}\right)\right\rangle,\left\langle\left(\triangle \hat{n}_{+}\right)^{2}\right)\right\rangle$ and $\left\langle\left(\triangle \hat{n}_{-}\right)^{2}\right\rangle$, respectively, for the given values of the interaction parameters. It is obvious that $\left.\left\langle\left(\triangle \hat{n}_{1}\right)^{2}\right)\right\rangle$ and $\left\langle\left(\triangle \hat{n}_{-}\right)^{2}\right\rangle$ exhibit RCP (see Fig. 8(a) and (c)) which is insensitive to the intensities of the initial 

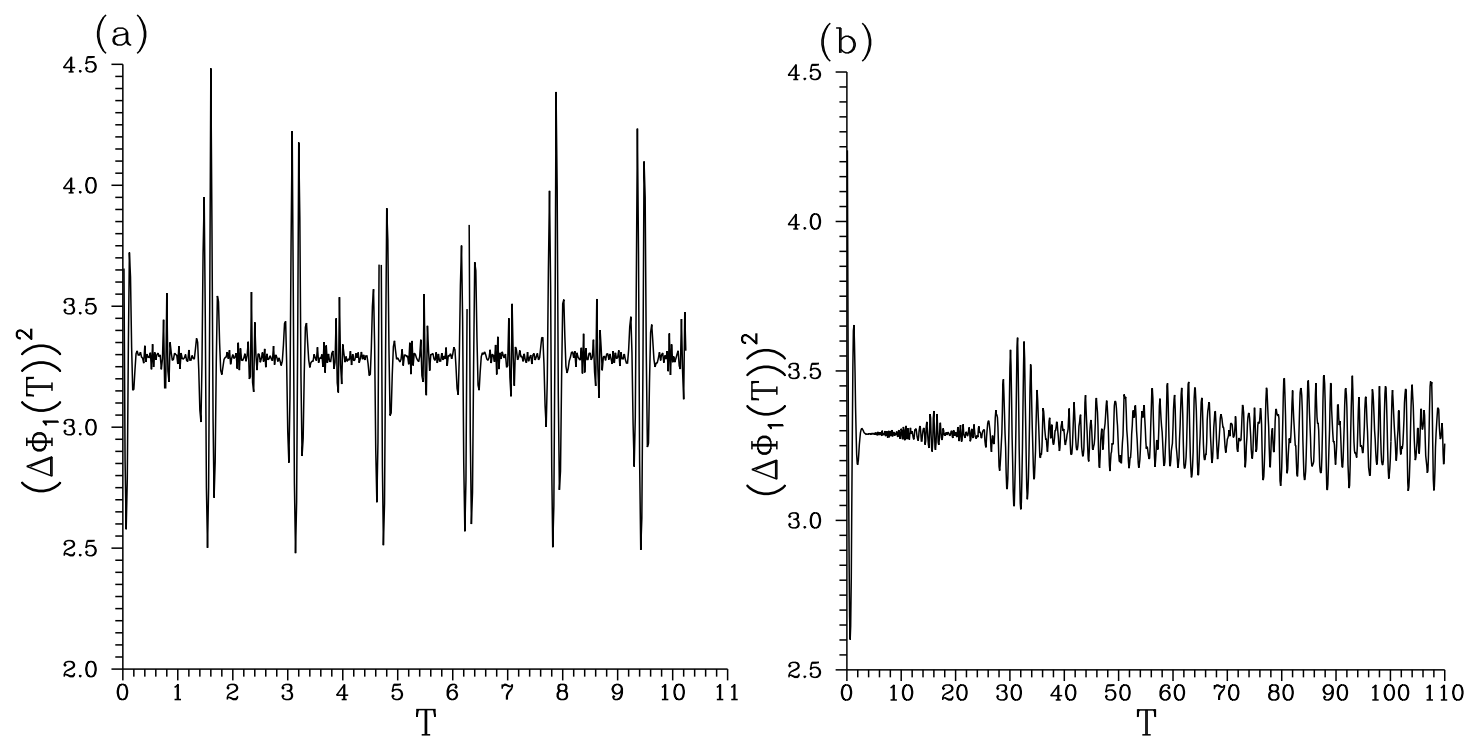

FIG. 7: The single-mode phase variance $\left\langle\left(\triangle \hat{\Phi}_{1}\right)^{2}\right\rangle$ of the first mode versus the scaled time $T$ for $\left|\alpha_{j}\right|=$ $5, \epsilon_{j}=1, \varphi=0, \phi=0,\left(k_{1}, k_{2}\right)=(2,2)(\mathrm{a})$ and $(1,2)(\mathrm{b})$.
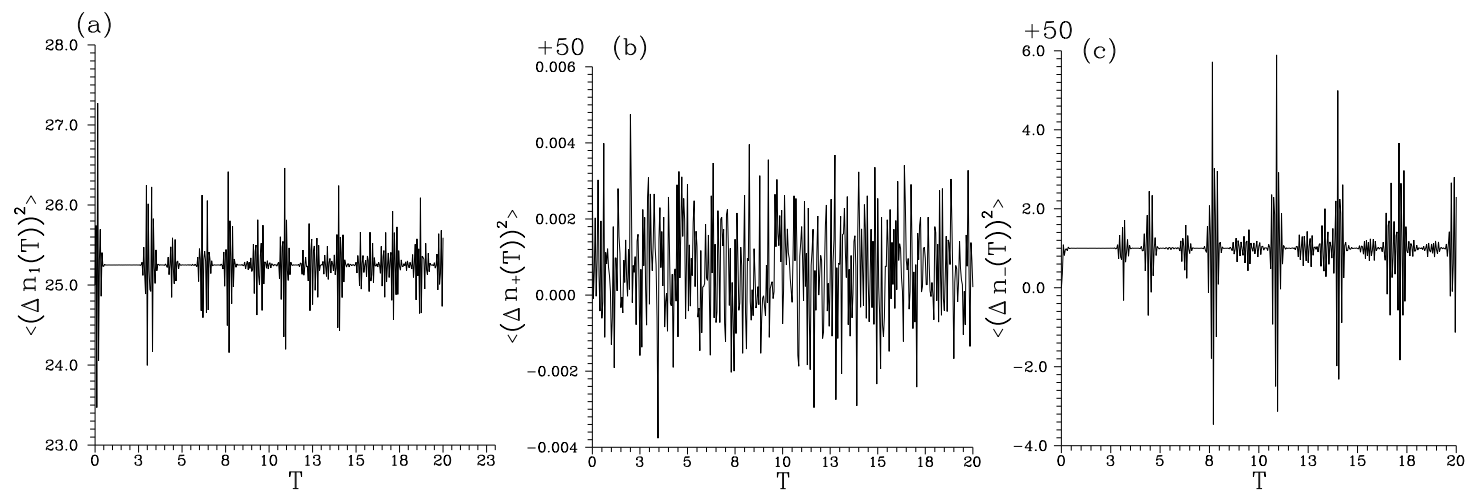

FIG. 8: The mean-photon number variances versus the scaled time $T$ for $\left|\alpha_{j}\right|=5, \epsilon_{j}=1, \varphi=0, \phi=$ $0,\left(k_{1}, k_{2}\right)=(1,1)$ and for the single-mode case (a), sum-mode case (b) and difference-mode case (c).

fields. Moreover, this behavior is almost similar to that of the corresponding atomic inversion. In Fig. 8(b) we can see that the chaotic behavior is dominant. To be more specific, the behavior of $\left\langle\left(\triangle \hat{n}_{+}\right)^{2}\right\rangle$ is close to the steady state, $\left.\left.\left\langle\left(\triangle \hat{n}_{+}(T)\right)^{2}\right)\right\rangle \simeq\left\langle\left(\triangle \hat{n}_{+}(0)\right)^{2}\right)\right\rangle$. Comparison between Fig. 8(b) and Fig. 8(c) shows that the correlation term, i.e. $\left\langle\hat{n}_{1} \hat{n}_{2}\right\rangle-\left\langle\hat{n}_{1}\right\rangle\left\langle\hat{n}_{2}\right\rangle$, plays a constructive (destructive) role in the behavior of the $\left\langle\left(\triangle \hat{n}_{-}\right)^{2}\right\rangle\left(\left\langle\left(\triangle \hat{n}_{+}\right)^{2}\right\rangle\right)$.

Now we can conclude that $\left\langle\left(\triangle \hat{\Phi}_{1}\right)^{2}\right\rangle$ can provide RCP, which is insensitive to the type of initial atomic state. We have to stress that the RCP in the atomic inversion and in the phase variances are completely different and cannot be compared. Finally, it is worth mentioning that RCP has been realised also for the phase variances of the two-photon down-conversion with quantized pump, 
e.g. [45].

\section{CONCLUSIONS}

In this paper we have discussed the phase properties of the lossless multiphoton two-mode Jaynes-Cummings model for Schrödinger-cat states in the framework of Pegg-Barnett formalism. The investigation has included also the dynamical behavior of the $W$ function at the phase space origin. We have shown that the evolution of the $\left\langle\hat{\sigma}_{z}(T)\right\rangle$ of the TMJCM is reflected in the behavior of the phase distribution where the splittings and overlappings of the phase distribution are accompanied by the collapses and revivals, respectively, in $\left\langle\hat{\sigma}_{z}(T)\right\rangle$. This has been remarked in the behavior of both the single-mode and compound-mode phase distributions. Furthermore, in the course of the secondary revival in $\left\langle\hat{\sigma}_{z}(T)\right\rangle$ the phase distribution exhibits behavior almost similar to that of the collapse case. Moreover, the nonclassical multipeak structure, which is representative to cat states, is remarked in the behavior of the phase distribution. Nevertheless, this behavior is insensitive to the type of both the initial cat state (, i.e. if the modes are initially prepared in even or odd coherent states) and the initial atomic states (, i.e. if it is in the excited or ground state). Additionally, we have shown that for higher-order photon transition, i.e. $k_{j}>1$, the phase distribution is in the steady state showing multipeak structure. Also we have proved that under certain condition there is a clear relationship between the atomic inversion and $W$ function. Such relation leads to the fact: There is a relationship between the $W(0, T)$ and the corresponding phase distribution. Finally, we have shown that the single-mode phase variances can exhibit RCP about the long-time interaction.

\section{Acknowledgement}

One of the authors (F.A.A.E.) is grateful to the International Islamic University Malaysia for hospitality and financial support.

\section{References}

[1] Jaynes E T and Cummings F W 1963 Proc. IEEE 5189. 
[2] Eberly J H, Narozhny N B and Sanchez-Mondragon J J 1980 Phys. Rev. Lett. 44 1323; Narozhny N B, Sanchez-Mondragon J J and Eberly J H 1981 Phys. Rev. A 23 236; Yoo H I, Sanchez-Mondragon J J and Eberly J H 1981 J. Phys. A 14 1383; Yoo H I and Eberly J H 1981 Phys. Rep. 118239.

[3] Meystre P and Zubairy M S 1982 Phys. Lett. A 89A 390.

[4] Kim M S 1993 J. Mod. Opt. 401331.

[5] Garraway B M, Sherman B, Moya-Cessa H, Knight P L and Kurizki G 1994 Phys. Rev. A 49 535; Kozhekin A, Kurizki G and Sherman B 1996 Phys. Rev. A 543535.

[6] Shore B W and Knight P L 1993 J. Mod. Opt. 401195.

[7] Buck B and Sukumar C V 1981 Phys. Lett. A 81 132; Singh S 1982 Phys. Rev. A 25 3206; Abdalla M S, Ahmed M M A and Obada A-S F 1990 Physica A 162 215, and references therein.

[8] Cardimona D A, Sharma M P and Ortega M A 1989 J. Phys. B 22 4029; Cardimona D A 1990 Phys. Rev. A 415016.

[9] Barnett S M and Knight P L 1984 Opt. Acta 31 435; ibid 1203; Mahmood S and Zubairy M S 1987 Phys. Rev. A 35 425; Iqbal M S, Mahmood S, Razmi M S K and Zubairy M S 1988 Opt. Soc. Am. B 5 1312; Sharma M P, Cardimona D A and Gavrielides A 1989 Opt. Commun. 72 291; Sharma M P, Cardimona D A and Gavrielides A 1989 J. Opt. Soc. Am. B 61942.

[10] Barnett S M and Knight P L 1986 Phys. Rev. A 22 2444; Puri R R and Agarwal G S 1986 Phys. Rev. A 33 3610; ibid 1987353433.

[11] Goy P, Raimond J M, Gross M and Haroche S 1983 Phys. Rev. Lett. 50 1903; Meshede D, Walther H and Muller G 1985 Phys. Rev. Lett. 54551.

[12] Burne M, Raimond J M and Haroche S 1987 Phys. Rev. A 35 154; Ashraf I and Zubairy M S 1990 Opt. Commun. 7785 .

[13] Rempe G, Walther H and Klein N 1987 Phys. Rev. Lett. 57353.

[14] Meekhof D M, Monroe C, King B E, Itano W M and Wineland D J 1996 Phys. Rev. Lett. 76 1796; Leibfried D, Meekhof D M, King B E, Monroe C, Itano W M and Wineland D J 1996 Phys. Rev. Lett. 77 4281; Monroe C, Meekhof D M, King B E, Wineland D J 1996 Science 272 1131; Leibfried D, Meekhof D M, Monroe C, King B E, Itano W M and Wineland D J 1997 J. Mod. Opt. 442485.

[15] Song S, Caves C M and Yurke B 1990 Phys. Rev. A 41 5261; Yurke B, Schleich W and Walls D F 1990 Phys. Rev. A 421703.

[16] Schrödinger E 1935 Nature 23844.

[17] El-Orany F A A, Peřina J, Peřinová V and Abdalla M S 2002 J. Opt. B: Quant. Semiclass. Opt. 5 60; ibid 2003 J. Europ. Phys. D 22141.

[18] Vidiella-Barranco A, Moya-Cessa H and Bužek V 1992 J. Mod. Opt. 391441.

[19] Gerry C C and Hach III E E 1993 Phys. Lett. A 1791.

[20] Joshi A and Singh M 1995 J. Mod. Opt. 42775.

[21] El-Orany F A A and Obada A-S 2003 J. Opt. B: Quant. Semiclass. Opt. 560.

[22] Barnett S M and Pegg D T 1986 J. Phys. A: Gen. Math. 19 3849; Pegg D T and Barnett M S 1988 
Europhys. Lett. 6 483; ibid 1988 J. Mod. Opt. 36 7; ibid 1989 Phys. Rev. A 391665.

[23] Dung H T, Tanaś R and Shumovsky A S 1990 Opt. Commun. 79462.

[24] Meng H X, Chai C L and Zhang Z M 1992 Phys. Rev. A 452131.

[25] Fan A-f 1993 Opt. Commun. 98 340; ibid 1994 Phys. Rev. A 491509.

[26] Abdel-Aty M, Abdalla M S and Obada A-S F 2002 J. Opt. B: Quant. Semiclass. Opt. 4 S133.

[27] Ekert A and Jozsa R 1996 Rev. Mod. Phys. 68 733; Lo H-K, Popescu S and Spiller T 1998 Introduction to Quantum Computation and Information (Singapore: World Scientific); Begie A, Braun D, Tregenna B and Knight P L 2000 Phys. Rev. Lett. 851762.

[28] Wheeler J A and Zurek W H 1983 Quantum Theory of Measurement (Princeton, NJ: Princeton University Press).

[29] Smithey D T, Beck M, Cooper J and Raymer M G 1993 Phys. Rev. A 48 3159; Beck M, Smithey D T and Raymer M G 1993 Phys. Rev. A 48 890; Beck M, Smithey D T, Cooper J and Raymer M G 1993 Opt. Lett. 18 1259; Smithey D T, Beck M, Cooper J, Raymer M G and Faridani M B A 1993 Phys. Scr. T 4835.

[30] Rosenhouse-Dantsker A 1992 J. Mod. Opt. 391471.

[31] Gerry C C and Eberly J H 1990 Phys. Rev. A 426805.

[32] Cardimona D A, Kovanis V, Sharma M P and Gavrielides A 1991 Phys. Rev. A 433710.

[33] Zaheer K and Zubairy M S 1988 Phys. Rev. A 371628.

[34] Phoenix S J D 1989 J. Mod. Opt. 36 1163; Peng J-S and Li G-X 1993 Phys. Rev. A 473167.

[35] Tanaś R, Miranowicz A and Gantsog Ts 1996 Progress in Optics ed. Wolf E Vol. 35 355; V. Peřinová, A. Lukš and J. Peřina 1998 Phase in Optics (Singapore: World Scientific).

[36] Banaszek K and Wódkiewicz k 1996 Phys. Rev. Lett. 76 4344; Wallentowitz S and Vogel W 1996 Phys. Rev. A $\mathbf{5 3} 4528$.

[37] Lutterbach L G and Davidovich L 1997 Phys. Rev. lett. 782547.

[38] Nogues G, Rauschenbeutel A, Osnaghi S, Bertet P, Brune M, Raimond J M, Haroche S, Lutterbach L G and Davidovich L 2000 Phys. Rev. A 62054101.

[39] El-Orany F A A 2004 J. Phys. A: Gen. Math. (Submitted).

[40] El-Orany F A A 2004 J. Phys. A: Gen. Math. 376157.

[41] Peřinová V 1981 Opt. Act. 28747.

[42] Peřinová V and Peřina J 1981 Opt. Act. 28769.

[43] Bužek V, Gantsog Ts and Kim M S 1993 Phys. Scr. A T48 131.

[44] Zaheer K and Zubairy M S 1989 Phys. Rev. A 392000.

[45] Gantsog Ts 1992 Phys. Lett. A 170 249; Gantsog Ts, Tanaś R and Zawodny R 1992 Act. Phys. Slov. 4374. 